Session 4

Stellar Cycles 


\title{
Stellar cycles: general properties and future directions
}

\author{
Mark S. Giampapa \\ National Solar Observatory \\ 950 N. Cherry Ave., POB 26732, Tucson, AZ 85726-6732 USA \\ email: giampapa@nso.edu
}

\begin{abstract}
We discuss the general properties of stellar cycles with emphasis on their amplitudes as a function of stellar parameters, particularly those stellar characteristics relevant to dynamodriven magnetic activity. We deduce an empirical scaling relation between cycle frequency and differential rotation based on previously established empirical relations. We also compare the recent Cycle 23 to cycles in solar-type stars. We find that the extended minimum of Cycle 23 resembled in its Ca II H \& K emission at minimum the mean levels of activity seen in stars with no cycles.
\end{abstract}

Keywords. Stars:activity cycle, Sun:cycle, stars:chromosphere, stars:differential rotation

\section{Introduction}

Any review of stellar cycles must begin with a reference to the pioneering study of Olin Wilson, who asked the question, "Does the chromospheric activity of main-sequence stars vary with time, and if so, how?" (Wilson 1978). The question was clearly inspired by the threefold recognition of (1) the existence of the solar cycle as most prominently observed in the form of the $\sim 11$-year variation in sunspot numbers, (2) the spatial association of chromospheric Ca II resonance line emission with magnetic field regions, on the Sun, particularly near spots, and (3) the observation of Ca II H \& K line emission in the spectra of late-type stars indicative of the presence of chromospheres, analogous to the solar chromosphere, in the outer atmospheres of stars. As we know from Wilson's dedicated efforts, and the long-term extension of this program by Sallie Baliunas and her colleagues at Mt. Wilson (Baliunas et al. 1995), late-type stars exhibit an array of chromospheric variability ranging from regular variations with multi-year periods similar to that of the Sun to irregular variability with no clear pattern to stars with essentially flat curves, i.e., no long-term cycle-like variability.

About $25 \%$ of the Mt. Wilson sample exhibits irregular variations with considerable scatter in $\mathrm{H} \& \mathrm{~K}$ core emission although some of these "irregular stars" can display aperiodic variability. Typically, these objects are characterized by relatively higher mean levels of Ca II emission compared to the Sun and they generally belong to a somewhat younger stellar population, such as members of the $\sim 600$ Myr Hyades. Approximately $60 \%$ of the Mt. Wilson sample shows smooth cyclic variability similar to that of the Sun. These stars also have mean levels of Ca II emission more near the mean level of the Sun. Finally, about $15 \%$ of the sample objects are simply constant, or 'flat', in their time series of $\mathrm{H} \& \mathrm{~K}$ emission suggesting either no cycle variability or a very long period cycle. While it might be expected that this group of stars would be characterized by an even lower mean level of Ca II chromospheric emission, continuing the implied trend, we will see that the 'flat case' is more complicated. 
In the following, I will further review the Mt. Wilson survey results in the context of correlations, beginning with cycle amplitudes and stellar properties and continuing with empirical relationships between cycle periods, rotation, and differential rotation. A comparison of the Sun with other solar-type stars in the context of different stellar samples will be discussed as will the most recent observations of new cycle properties, such as multiple cycles and short-period cycles. The possible implications for dynamo models will be noted but without firm conclusions. The review concludes with a summary followed by a discussion of promising directions for future research.

\section{Amplitudes of Stellar Cycles}

We refer the reader to Fig. 2 in Saar \& Brandenburg (2002), which gives the amplitude of stellar cycles in dwarf stars as the fractional change in the normalized chromospheric flux in the $\mathrm{Ca}$ II $\mathrm{H} \& \mathrm{~K}$ resonance lines relative to the mean level of this index, as a function of $B-V$ color, or stellar effective temperature. Inspection of their Fig. 2 reveals that relative cycle amplitudes generally increase toward cooler stars with thicker convection zones (as a fraction of the stellar radius), attaining an apparent maximum in the mid-K spectral types. Thereafter, relative amplitudes decline though it is important to note that the regime of the $\mathrm{M}$ dwarf stars has not been extensively explored. The reason for this is primarily observational given the comparative faintness of these cool stars combined with the reduced efficiencies of detectors in the blue/near-UV region of the $\mathrm{H} \& \mathrm{~K}$ lines near $400 \mathrm{~nm}$.

Saar \& Brandenburg (2002) find that cycle amplitudes do not exhibit a strong dependence on rotation or, in the case they considered, inverse Rossby number. However, we know from the rotation-activity connection that chromospheric emission does depend on rotation (e.g., Noyes et al. 1984). Therefore, the change in chromospheric emission as modulated by the cycle must behave in a similar way on rotation as does the mean level of emission. Since the fractional change in the chromospheric emission index, i.e., the amplitude of the cycle, is always less than one, Saar \& Brandenburg (2002) suggest that only a portion of the mean $\mathrm{H} \& \mathrm{~K}$ emission derives from a cycling dynamo, i.e., the "cycling portion" modulates the mean magnetic field-related emission arising from the non-cycling (small-scale) component of the dynamo, which must have a similar dependence on rotation as the larger-scale, cycling component.

We refer the reader, again from Saar \& Brandenburg (2002), to their Fig. 4, where the observed relationship between two dimensionless quantities representing the logarithm of cycle amplitude versus the logarithm of the cycle frequency divided by the rotation frequency. Representing the data in this way reveals two relations, or "branches" - the "A" or "Active" branch and the "I" or "Inactive" branch. While a single relation using dimensional quantities can be constructed to fit the data (Baliunas et al. 1996), it has considerably more scatter than the representation in Fig. 4 of Saar \& Brandenburg (2002). The other appeal is that the presence of the two branches suggests a discontinuity or jump between "active" and "quiet" stars that is reminiscent of the so-called Vaughan-Preston gap (Vaughan \& Preston 1980). In this kind of "Chromospheric H-R Diagram" there is a distinctive gap separating active (and generally younger) stars from more quiescent and generally older stars. One prevailing thought as to the origin of the Vaughan-Preston gap is that it is the manifestation of two different dynamo modes. Another minority view is that the gap is the result of a discontinuity in the local star formation rate combined with the assumption of a smooth decline of chromospheric activity with age.

Saar \& Brandenburg (1999) offer an interpretation in the context of $\alpha-\omega$ kinematic dynamo models where the $\alpha$ effect (i.e., the poloidal flux generation) is sensitive to 
stellar rotation. Recalling briefly, this class of dynamo models requires a region of radial differential rotation where poloidal field lines interact with azimuthal flows to produce toroidal flux (the " $\omega$ effect"). The interaction of buoyant toroidal flux with cyclonic fluid motions in the convection zone produces poloidal fields that can then be acted upon again by the $\omega$ effect - this process is referred to as the " $\alpha$ effect." Saar \& Brandenburg (1999) consider the hypothesis that the dynamo is partly unstable, giving rise to a transition between modes that, in turn, results in the Inactive (I) and Active (A) branches, respectively. The mode transition itself may be due to rotation-dependent changes in the degree of enhancement of the $\alpha$ effect, presumably due to interactions of toroidal flux with rotation and fluid motions in the convection zone.

\section{Rotation, Differential Rotation, and Cycle Periods}

Given that rotation and differential rotation are key physical parameters in models for the origin of magnetic flux generation in the Sun and cool stars, their inter-relationships along with their relationship to cycle parameters, such as cycle period, merit consideration. In Fig. 1 (from Saar 2011), we see differential rotation frequency, normalized to solar differential rotation, as a function of rotation frequency. These kinds of data are difficult to obtain since they depend on the detection of subtle period changes in long-term observations of the rotational modulation of active regions on the stellar surface. In Fig. 1 the slower rotators follow a power law with normalized $\Delta \Omega \sim \Omega^{0.68}$ for $\Omega<3 \mathrm{~d}^{-1}$. The relation appears to reach a maximum and then declines toward rapid rotators that also are "saturated activity" stars, i.e., stars characterized by high levels of chromospheric and coronal emission levels that do not increase further with faster rotation rates. Their surfaces are thought to be completely covered, or "saturated," with magnetic active regions. However, if these objects are plotted in a graph of differential rotation versus $B-V$ color then a clear trend of declining differential rotation with redder $B-V$ color is revealed (Saar 2011, his Fig. 1). The result suggests a dependence of differential rotation on effective temperature or, in turn, relative convection zone depth, which implies a possible relationship with convective turnover times. This immediately evokes the Rossby number (or inverse Rossby number) as a relevant parameter since the Rossby number incorporates both rotation period and convective turnover time in a single

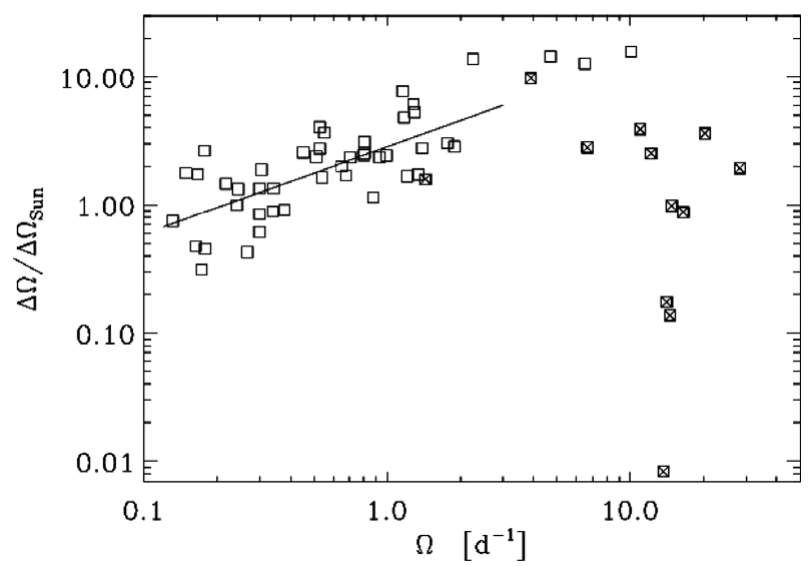

Figure 1. Normalized differential rotation frequency versus rotation frequency (from Saar 2011) 

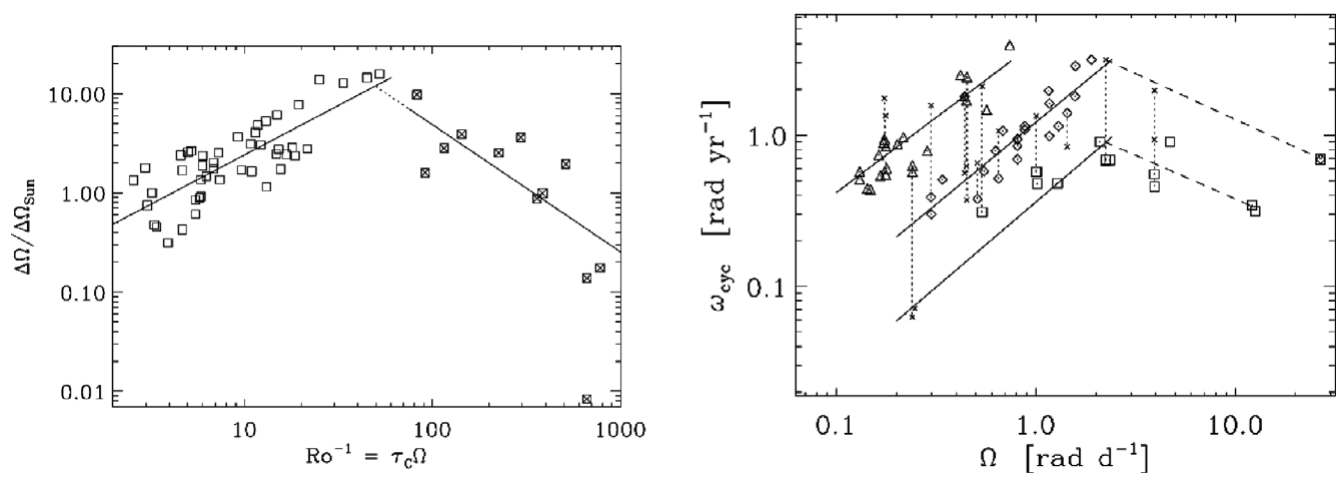

Figure 2. A comparison of the behavior of cycle frequency with inverse Rossby number and normalized differential rotation frequency with rotation (from Saar 2011)

dimensionless quantity. Saar (2011) points out that this suggests a relationship involving deep-seated dynamo action at rapid rotation in the especially high-activity stars.

When cycle frequency is examined as a function of rotation frequency, three parallel tracks emerge in the data, each with cycle frequency, $\omega_{c y c} \sim \Omega^{1.1}$ (Saar 2011). We compare this relation to that for normalized differential rotation but now as a function of inverse Rossby number rather than just rotation frequency (Fig. 2). The cycle frequency - rotation frequency diagram exhibits a rise to a peak followed by a decline though the decline is somewhat tentative given the scant data available. Nevertheless, stars with well-defined cycle periods show a correlation with rotation. This diagram appears very similar in shape and in location of the maximum to that in the differential rotation versus inverse Rossby number diagram. Saar (2011) claims that the similarity in shape of these relations suggests that differential rotation has left an "imprint" on cycle periods and their relationship to rotation. From an observational perspective, the way in which we measure differential rotation and cycle periods, i.e., varying concentrations of magnetic flux in time and latitude/longitude distribution on stars, could naturally lead to similar diagrams. In particular, detecting differential rotation in late-type stars requires the appearance of active complexes at different latitudes and asymmetrically distributed in longitude in order to see rotational modulation. Cycle modulation requires a globally averaged variation in chromospheric $\mathrm{H} \& \mathrm{~K}$ emission over long periods of time, which may be correlated with the latitudinal variation in the appearance of active region complexes over cycle time scales. In other words, the similarity of these two diagrams could be evidence for a "butterfly diagram" in stars, analogous to what we see in the Sun, where emergent sites of active complexes move from high-latitudes (slower differential rotation) to lower latitudes (faster differential rotation) over the course of a cycle.

Recalling our discussion of cycle amplitudes, and the empirical relationships given above between rotation, differential rotation, and cycle frequency, we can examine further the dependence of cycle amplitudes on these parameters. In particular, Saar \& Brandenburg (2002) find that cycle amplitude, $A_{c y c}$, is proportional to $\left(\omega_{c y c} / \Omega\right)^{\alpha}$, where $\alpha$ is 0.65 for the I-branch and 0.85 for the A-branch stars, respectively. We recall that $\omega_{c y c} \sim \Omega^{1.1}$. Hence, by substitution, $A_{c y c} \sim \Omega^{0.1 \alpha}$. Thus, cycle amplitudes have a very weak dependence on rotation, as noted earlier by Saar \& Brandenburg (2002; see their Fig. 3) from observations. We may explore the correlation of cycle amplitudes with differential rotation given the power-law relation between normalized differential rotation and rotation frequency cited previously. Again we find that cycle amplitudes depend only weakly on differential rotation with $A_{c y c} \sim\left(\Delta \Omega / \Delta \Omega_{\odot}\right)^{0.1}$, for either the 
I-branch or the A-branch. By contrast, we can use the aforementioned empirical relationships between differential rotation and rotation, and cycle frequency and rotation, to find $\omega_{c y c} \sim\left(\Delta \Omega / \Delta \Omega_{\odot}\right)^{1.6}$. Hence, a star with twice the differential rotation frequency of the solar value would have a cycle frequency approximately three times higher and, therefore, a correspondingly shorter period.

\section{The Sun in a Stellar Context}

Recent observational work has expanded in different ways on the original Mt. Wilson program using stellar samples that either overlap or are completely different from the Mt. Wilson sample. In a recent preprint, Lovis et al. (2011) utilized data from the HARPS (High-Accuracy Radial velocity Planetary Searcher) survey-intended for Doppler searches for extrasolar planets - to examine the systematic chromospheric properties in their subsample, including possible magnetic cycle detection during the $\sim 7$ years the survey has been in operation. Their subsample includes stars that are broadly solar-type that also were selected for their low projected rotation velocities of $\mathrm{v}$ $\sin i<3-4 \mathrm{~km}_{-} \mathrm{s}^{-1}$ and single status (i.e., no evidence the star is a member of a binary or multiple system).

An overview of the results from the subsample analyzed by Lovis et al. (2011; see their Fig. 14) are encapsulated in Fig. 3 showing the mean chromospheric emission index as a function of effective temperature, i.e., a chromospheric "H-R diagram" referred to earlier in the discussion of the Vaughan-Preston gap in Section 2. Please see the electronic version of this paper for the color-coded version of Fig. 3 discussed in the following, or Figs. 14 and 13, respectively, in Lovis et al. (2011). Stars with "large-amplitude" cycles are represented by red filled circles while stars with no large-amplitude cycle, to within their adopted criterion of a relative variation of $4 \%$, are denoted by the blue dots. The black filled circles represent stars with no detected cycle but for which the existence of a cycle cannot be completely ruled out. The Sun is included at its mean value for the chromospheric index, among other stars with detected cycles. Interestingly, and perhaps suggestively, the mean Sun also appears in this diagram to reside very near a transition between stars with cycles and stars with no cycles.

The H-R diagram of the subsample studied by Lovis et al. (2011; their Fig. 13), given here as Fig. 4, yields further insight on a comparison of the two populations of stars with cycles and without 'large-amplitude' cycles. Lovis et al. (2011) note that the stars without cycles appear among the warmer stars and do not appear among the cooler $\mathrm{K}$ dwarfs. While this is indeed true, the salient point is that the stars without cycles are systematically slightly higher in luminosity (at a given effective temperature), suggesting that some evolution has occurred, i.e., they belong to a slightly older population. The fact that it is the warmer stars, which are also the relatively more massive stars, is consistent with membership in a slightly older population since they will evolve faster than the cooler dwarfs. Furthermore, rotational evolution in the form of spin-down will occur more rapidly among the more massive stars (Stauffer et al. 1991).

It is worthwhile to consider the Sun and its cycle in the above context of cycling and non-cycling solar-type stars. We display in Fig. 5 the time series of the $0.1 \mathrm{~nm} \mathrm{K-index}$ parameter from the SOLIS Integrated Sunlight Spectrometer (ISS) of the National Solar Observatory at Kitt Peak.

The ISS obtains spectra of the Sun seen as a star. The K-index is a measure of the strength of the core of the Ca II K line, which includes the chromospheric emission. From inspection of the SOLIS ISS K-index parameter time series we find that the Kindex minimum for Cycle 23 occurred during June 2008. We have the same time series 


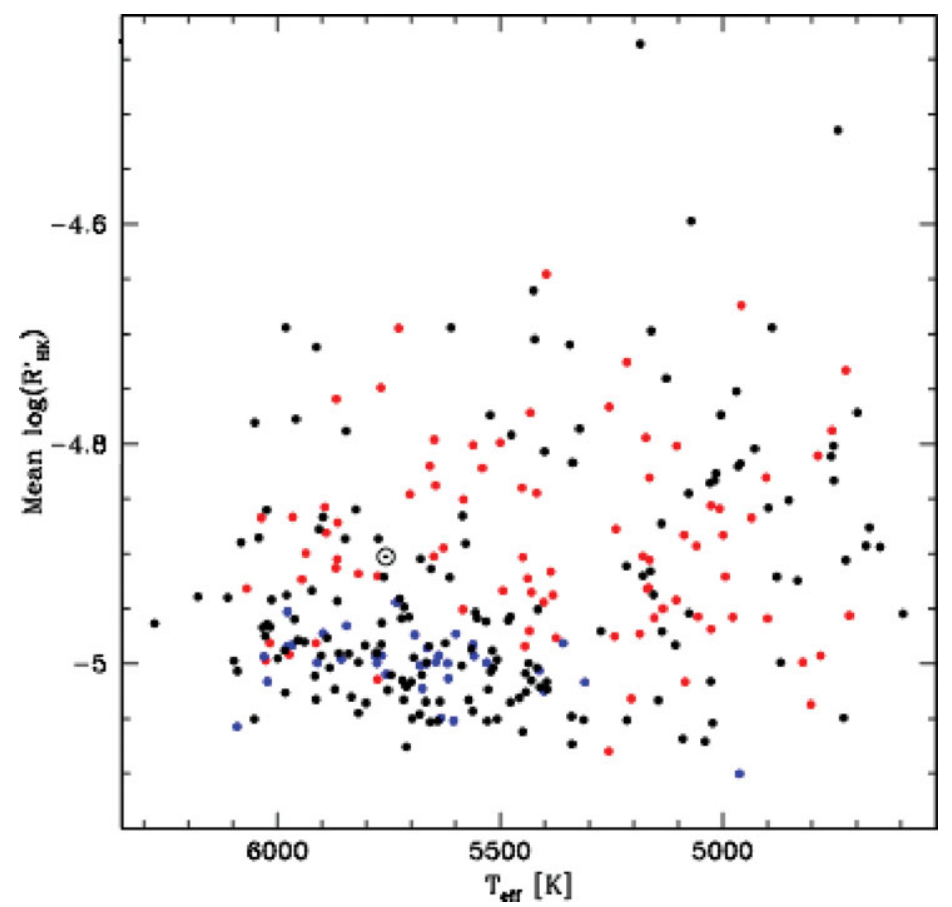

Figure 3. Normalized mean chromospheric emission for stars with various cycle properties as a function of effective temperature (from Lovis et al. 2011)

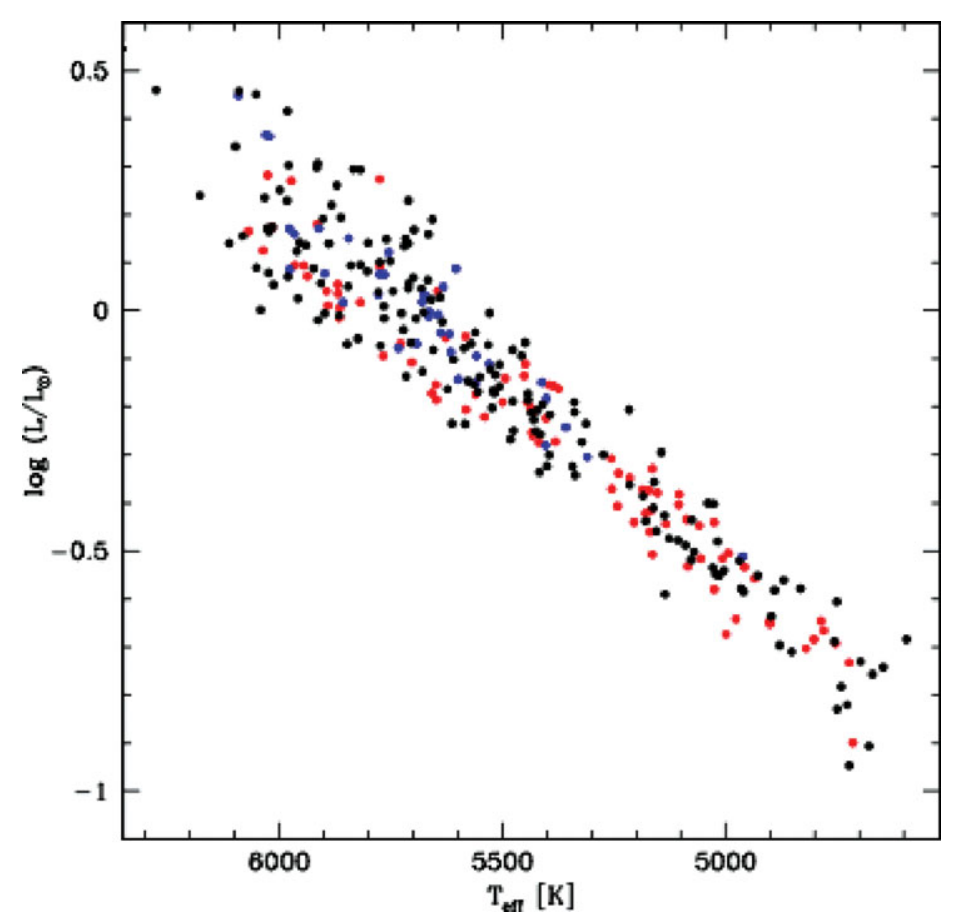

Figure 4. H-R diagram of HARPS subsample of solar-type stars (from Lovis et al. 2011) 


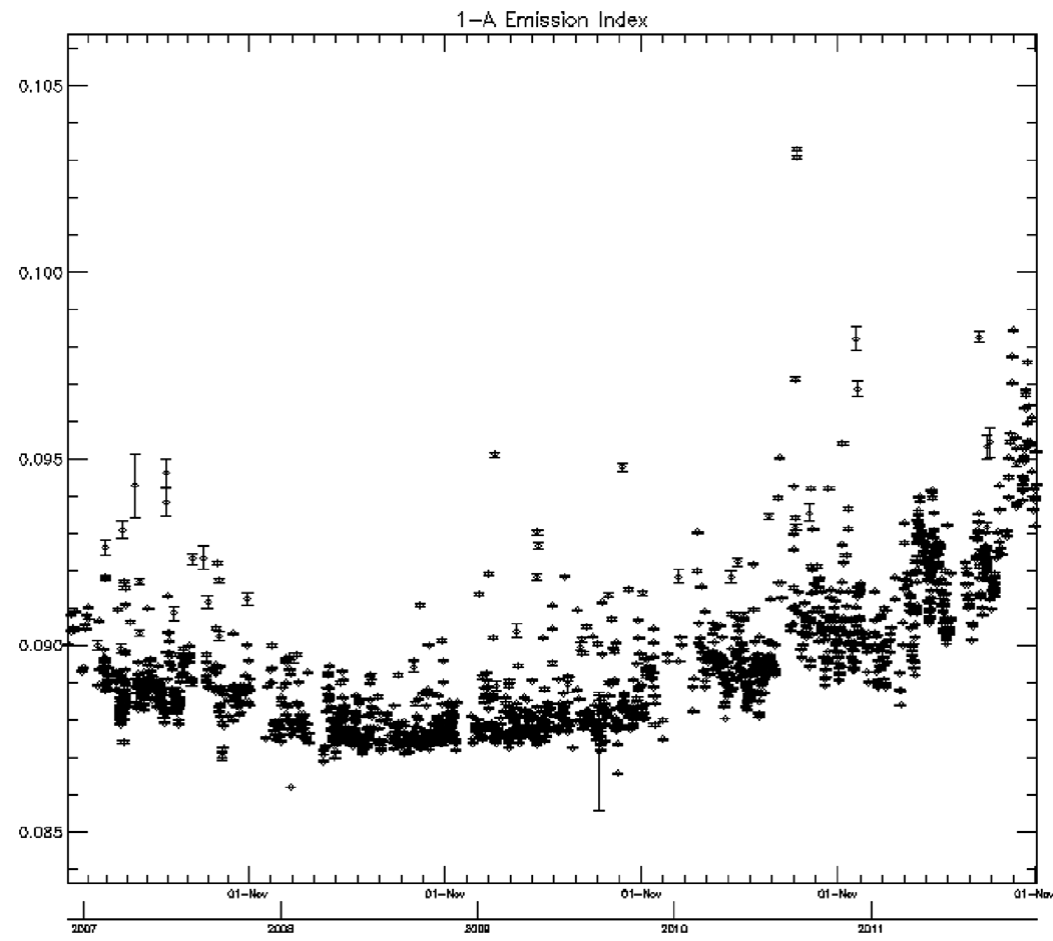

Figure 5. Time series of the Ca II K line $1.0 \AA$ index from the SOLIS ISS instrument

for the Ca II $\mathrm{H}$ line. This enables us to combine the $\mathrm{H}$ and $\mathrm{K}$ series to derive the solar counterpart of the stellar HK index - a combined measure of the H \& $\mathrm{K}$ line core strength - following a calibration procedure described by Giampapa et al. (2006). The resulting value of the corresponding stellar $\mathrm{R}_{H K}^{\prime}$ index for the Cycle 23 minimum Sun is $1 \times 10^{-5}$. Referring back to Fig. 3, this places the Sun seen as a star at its Cycle 23 minimum in the very midst of other stars observed to not have a large-amplitude cycle, as defined by Lovis et al. (2011). In other words, a distant observer performing a similar survey would have concluded that the Cycle 23 minimum Sun was a candidate for a star in a Maunder minimum state of prolonged quiescence. It is worthwhile to examine the Cycle 23 'minimum Sun' in comparison with other stellar samples.

In their survey of the chromospheric activity of the solar-type stars in the solar-age and solar-metallicity cluster, M67, by Giampapa et al. (2006), the position of the Sun at the 2008 minimum in their Fig. 3 would be among the most quiet sun-like stars in M67, below the previous recorded solar minima. It is important to note that M67 includes a homogeneous sample of solar-type stars, in contrast to field star samples such as the HARPS sample, which tend to be inhomogeneous in age and chemical composition. We therefore may ask if the especially quiescent sun-like stars in M67 are in a Maunderminimum state or in a prelude to a state of prolonged quiescence. Furthermore, is the Sun in a transition to a period of prolonged quiescence, perhaps even another Maunder Minimum?

A stellar candidate for such a transition to a Maunder-minimum-like state is HD 3851 from Baliunas et al. (1995), displayed in Fig. 6. The star is cooler than the Sun with a spectral type between $\mathrm{K} 0$ and $\mathrm{K} 2$, and with a cycle period of nearly 14 years. The amplitude of variation has declined significantly. In addition, there appears to be a longterm trend of a declining mean level of, in this case, the Mt. Wilson S-index, which is a 


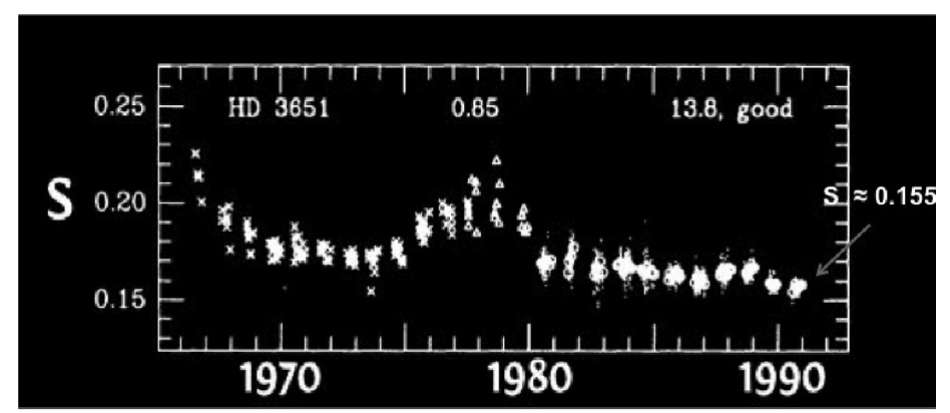

Figure 6. Example of a star that may be in transition to a Maunder-minimum state. The figure is provided courtesy of J. Hall and adapted from Baliunas et al. (1995)

measure of the strength of the $\mathrm{H} \& \mathrm{~K}$ bands normalized to nearby continuum levels. As we saw previously (Fig. 3), low mean chromospheric emission is correlated with the lack of large-amplitude cycles. The low point shown in the last measurements of this object from the Mt. Wilson program is at $\mathrm{S}=0.155$. This corresponds to the same $\mathrm{S}$-index value at the Cycle 23 minimum Sun in 2008. We envision a Maunder-minimum state as one in which there is either no or only a low-amplitude cycle at a low mean level of activity. But to confuse this picture further, in their monitoring of 57 sun-like stars, Hall \& Lockwood (2004) classified ten stars as "flat" (over 6-10 years of observation of each target). Yet their mean level of chromospheric emission in the flat-activity stars spans the range of emission levels from below solar minimum values to well in excess of solar maximum levels. We note, parenthetically, that the respective distributions of $\mathrm{S}$ index values for the cycling and the non-cycling, flat stars in the Hall \& Lockwood (2004) sample are statistically identical in the sense that there are no differences in the population from which they are drawn. Thus, flat time series in $\mathrm{H} \& \mathrm{~K}$ are not always correlated with only low levels of activity. The distribution of HK values for the Hall \& Lockwood (2004) sample, and for the Giampapa et al. (2006) M67 solar-type stars, appears to be unimodal. There does not appear to be a bimodal distribution as was reported in the earlier study of Baliunas \& Jastrow (1990) that, as shown later by Wright (2004), was contaminated with evolved subgiant stars that were in a quiescent state as a consequence of their relative age.

\section{Other Facets of Stellar Cycles}

Baliunas et al. (1995) discovered examples of solar-type stars with multiple cycles. For example, 15 Sge (HD 190406; G1 V) appears to have a shorter, secondary cycle of 2.6 years superimposed on its much longer primary cycle of 16.9 years (also see Hall et al. 2007). In an investigation based on the results from the Mt. Wilson sample, Böhm-Vitense (2007) investigated the correlation between cycle periods and rotation periods, identifying both an "Active" (A) branch and an "Inactive" (I) branch (see her Fig. 1). Secondary cycles were found to be primarily on the extrapolation of the I-sequence to faster rotation periods. Böhm-Vitense (2007) therefore suggested that the operative dynamo for the I-sequence stars must also be operating in the A-branch stars, and that the secondary cycles arise from the same I-branch dynamo mechanism. The Sun in her diagram appears conspicuously between the two branches, thereby giving the impression that it is a "transition object." Perhaps so, but I would caution that in this sample the I-branch stars in her Fig. 1 are all K stars with slower rotation than the Sun. Therefore, this may not be a suitable sample for comparison with the Sun. This points to the critical 
importance of how a stellar sample is defined before conclusions can be drawn concerning the comparative behavior of the Sun with that of other stars.

In the case of the Sun, Fletcher et al. (2010) report the detection of frequency shifts in low-degree solar oscillation modes from BISON data, suggesting evidence of an $\sim 2$ year period that may be interpreted as a secondary cycle. Jain et al. (2011) could not confirm this claim using GONG data but the issue may still be outstanding pending a more detailed comparison of the data analysis methods utilized in the two respective studies. I would remark, however, that in my own analysis of the multi-decadal Ca II K line time series obtained by W. C. Livingston utilizing the NSO McMath-Pierce solar telescope, I could not find evidence for an $~ 1-2$ year period arising from a possible cycle secondary to the primary 11-year solar cycle. In brief summary, the presence of secondary cycles has been interpreted as evidence of more than one dynamo operating in solar-type stars and, perhaps, in the Sun itself. However, more than one mode of a single dynamo mechanism may also be operative and distinguishing between these possibilities is a current challenge for dynamo theory.

In addition to secondary cycles, unusually short period cycles have been identified. In particular, Metcalfe et al. (2010) reported the detection of a 1.6 year cycle in a F8 V star with no evidence for a long-term trend indicative of a longer cycle. Therefore, this short cycle could be its primary cycle. Recalling the empirical scaling between cycle frequency and differential rotation given in $\S 2$, such a short period could be a manifestation of particularly strong differential rotation at a level of $\sim 3$ times the solar differential rotation rate.

\section{Summary and Future Directions}

Some of the principal points I sought to convey to the reader include the following:

- Cycle amplitudes exhibit similar trends with cycle frequency (normalized by rotation frequency) for stars on the Active and Inactive branches, respectively. However, cycle amplitudes do not show any dependence on rotation frequency alone, or on differential rotation.

- Cycle frequencies have a relatively stronger correlation with differential rotation frequency than with rotation frequency. We deduced an empirical scaling relation between cycle frequency and differential rotation given by $\omega_{\text {cyc }} \sim\left(\Delta \Omega / \Delta \Omega_{\odot}\right)^{1.6}$

- The Sun appears to reside at a boundary between stars with and without cycles. In fact, the recent Cycle 23 "minimum Sun" seen as a star looked like stars with no cycles, i.e., a possible 'Maunder-minimum star.'

- Relatively short period secondary cycles superimposed on longer period primary cycles have been detected in other solar-type stars and may exist in the Sun itself. Most stars with secondary periods tend to have higher mean levels of chromospheric emission and faster rotation as compared to the Sun.

- The existence of secondary cycles, and an Active and Inactive branch in cycle frequency (period)-rotation frequency (period) diagrams, have been interpreted as evidence of more than one dynamo mechanism operating in at least some stars and perhaps the Sun itself. Alternatively, multiple cycle periods may be manifestations of multiple modes of a single dynamo mechanism.

As for future directions of research, the future is now as the application of asteroseismic techniques to the detection of stellar cycles or possible secondary solar cycles becomes more extensive. In addition, starspot cycles inferred from Kepler and CoRoT data will be invaluable. Both these approaches will depend critically on the long term continuation of ground-based facilities and these revolutionary space missions. This will require both 
diligence and vigilance with funding agencies to maintain these crucial capabilities. In parallel efforts, it is extremely important to identify solar twins and homogeneous populations of stars in order to carry out comparative studies of stellar cycles and the solar cycle. Inhomogeneous stellar samples can lead to erroneous conclusions. The solar-age and solar-metallicity open cluster, M67, appears to be an excellent solar laboratory with stars that are spectroscopically identical to the Sun (e.g., Önehag et al. 2011). Finally, the cycle properties in the limit of thick convection zones as could be investigated through long-term studies of $\mathrm{M}$ dwarf stars remains an open and urgent question.

\section{Acknowledgements}

I am pleased to acknowledge discussions with S. Saar and J. Hall that materially contributed to this manuscript. I also would like to thank the organizers of IAU Symposium 286 in Mendoza, Argentina for their kind hospitality and for a productive and thoroughly enjoyable meeting. The National Solar Observatory is operated by AURA for the U. S. National Science Foundation under a cooperative agreement.

\section{References}

Baliunas, S. L. et al. 1995, ApJ, 438, 269

Baliunas, S. L. et al. 1996, ApJ, 460, 848

Baliunas, S. L. \& Jastrow, R. J. 1990, Nature, 348, 520

Böhm-Vitense, E. 2007, ApJ, 657, 486

Giampapa, M. S. et al. 2006, ApJ, 651, 444

Hall, J. C. \& Lockwood, G. W. 2004, ApJ, 614, 942

Hall, J. C., Lockwood, G. W., \& Skiff, B. A. AJ, 133, 862

Fletcher, S. T. et al. 2010, ApJ (Letters), 718, L19

Jain, K. et al. 2011, ApJ, 739, 6

Lovis, C. et al. 2011, $A \& A$, submitted

Metcalfe, T. S. et al. 2010, ApJ (Letters), 723, L213

Noyes, R. W. et al. 1984, ApJ, 279, 763

Önehag, A. et al. 2011, A\&A, 528, 850

Saar, S. H. 2011, The Physics of Sun and Star Spots, Proceedings of IAU Symposium No. 273, eds. D. P. Choudhary \& K. G. Strassmeier, p. 61

Saar, S. H. \& Brandenburg, A. 1999, ApJ, 524, 295

Saar, S. H. \& Brandenburg, A. 2002, AN, 323, 357

Stauffer, J. R. et al. 1991, ApJ, 374, 142

Vaughan, A. H. \& Preston, G. W. 1980, PASP, 92, 385

Wilson, O. C. 1978, ApJ, 226, 379

Wright, J. T. 2004, AJ, 128, 1273

\section{Discussion}

Arnab Choudhuri: 1) You showed very interesting plots of differential rotators, are they all solar type with the equator moving faster or are there anti-solar cases in the sample? 2) You showed a star which was a candidate to enter a Maunder Minimum. The data went only until 1990, do you have data in the last 16 or 20 years?

MARK Giampapa: 1) The differential rotation is inferred by changes in rotation period coming from the rotational modulation of active regions asymmetrically distributed in longitude. So, there is no latitude information and, therefore, we don't know if there are anti-solar examples. 2) No, there are no subsequent data. The Mt. Wilson program ended due to lack of funding. 
EMRE IşIK: You said there was a flat activity star with a considerable HK flux but is not showing cycles. Are there more stars like this in the sample?

MARK GIAmPAPA: The sample of stars is from Jeff Hall's "S-cubed" monitoring program at Lowell Observatory in Flagstaff, Arizona. There are 57 solar-type stars in his sample, of these, 10 show 'flat activity', that is, no cycle-like or statistically significant variation.

LeIf SvalgaARD: During the Maunder Minimum we know from cosmic ray data that the dynamo was still going. If magnetic activity is still there, there should be Ca II emission going in a cyclic fashion with regard to your stellar cycling and non-cycling data. Do you have any comment?

Mark Giampapa: The detection of cycle variation depends on the index or diagnostic being used., whether it is sunspot number, modulation of cosmic rays, or something else. In the flat activity stars, the time series of cyclic Ca II emission is not present within the small errors. Of course, we don't have direct information on the integrated Ca II emission during the Maunder Minimum. So, it is difficult to know what the limits of Ca II variability might have been.

JEFF LinsKY: If we observe the Sun from the poles, the solar cycle would have looked less intense. Could it be that stars with low stellar cycles are seen from the poles?

MARK GIAMPAPA: In Hall's sample of 57 stars, there are 10 stars that exhibit flat activity in their K-line time series. Statistically, it is an extremely low probability that in a sample of only 57 stars, 10 would be seen nearly pole-on. Also, one could still see a cycle modulation of the K-line emission for a star viewed pole-on though the amplitude would be smaller.

Steve SAAR: (Comment) You have to be careful about what you use for your index, they have not been calibrated for effective metallicity or gravity because different metallicities or gravities can shift can shift the curve in the R'HK-effective temperature diagram, mainly due to changing the correction for the photospheric contribution to the $\mathrm{H}$ and $\mathrm{K}$ line cores.

MARK GIAmpapa: Yes, I agree. Lovis et al. in their paper do make a correction for metallicity to the R'HK index. 